\title{
Local Activity Determines Functional Connectivity in the Resting Human Brain: A Simultaneous FDG-PET/fMRI Study
}

\author{
Valentin Riedl, ${ }^{1,2,4}$ Katarzyna Bienkowska, ${ }^{1,4}$ Carola Strobel,,${ }^{1,4}$ Masoud Tahmasian, ${ }^{1,2,4}$ Timo Grimmer, ${ }^{3,4}$ \\ Stefan Förster, ${ }^{2,4}$ Karl J. Friston, ${ }^{5}$ Christian Sorg, ${ }^{1,3,4 *}$ and Alexander Drzezga ${ }^{6 *}$ \\ Departments of ${ }^{1}$ Neuroradiology, ${ }^{2}$ Nuclear Medicine, and ${ }^{3}$ Psychiatry, and ${ }^{4}$ Technische Universität München-Neuroimaging Center, Klinikum Rechts der \\ Isar der Technischen Universität München, 81675 München, Germany, ${ }^{5}$ The Wellcome Trust Centre for Neuroimaging, University College London, London \\ WC1N 3BG, United Kingdom, and ${ }^{6}$ Department of Nuclear Medicine, Uniklinik Köln, 50937 Köln, Germany
}

Over the last decade, synchronized resting-state fluctuations of blood oxygenation level-dependent (BOLD) signals between remote brain areas [so-called BOLD resting-state functional connectivity (rs-FC)] have gained enormous relevance in systems and clinical neuroscience. However, the neural underpinnings of rs-FC are still incompletely understood. Using simultaneous positron emission tomography/ magnetic resonance imaging we here directly investigated the relationship between rs-FC and local neuronal activity in humans. Computational models suggest a mechanistic link between the dynamics of local neuronal activity and the functional coupling among distributed brain regions. Therefore, we hypothesized that the local activity (LA) of a region at rest determines its rs-FC. To test this hypothesis, we simultaneously measured both LA (glucose metabolism) and rs-FC (via synchronized BOLD fluctuations) during conditions of eyes closed or eyes open. During eyes open, LA increased in the visual system, and the salience network (i.e., cingulate and insular cortices) and the pattern of elevated LA coincided almost exactly with the spatial pattern of increased rs-FC. Specifically, the voxelwise regional profile of LA in these areas strongly correlated with the regional pattern of rs-FC among the same regions (e.g., LA in primary visual cortex accounts for $\sim 50 \%$, and LA in anterior cingulate accounts for $\sim 20 \%$ of rs-FC with the visual system). These data provide the first direct evidence in humans that local neuronal activity determines BOLD FC at rest. Beyond its relevance for the neuronal basis of coherent BOLD signal fluctuations, our procedure may translate into clinical research particularly to investigate potentially aberrant links between local dynamics and remote functional coupling in patients with neuropsychiatric disorders.

Key words: energy metabolism; functional connectivity; PET/MR imaging

\section{Introduction}

Recent functional magnetic resonance imaging (fMRI) revealed organized activity in the brain at rest, an idea that has gained enormous relevance for systems and clinical neuroscience (Fox and Raichle, 2007; Greicius, 2008; Bressler and Menon, 2010; Menon, 2011). Particularly, this organized activity is defined by synchronous, low-frequency $(<0.1 \mathrm{~Hz})$ fluctuations of the blood oxygenation level-dependent (BOLD) fMRI signal between remote brain areas, termed resting-state functional connectivity (rs-FC). However, the neurophysiological and metabolic under-

Received Feb. 4, 2014; revised March 13, 2014; accepted March 27, 2014.

Author contributions: V.R., C. Sorg, and A.D. designed research; V.R., K.B., C. Strobel, T.G., and S.F. performed research; V.R. contributed unpublished reagents/analytic tools; V.R., K.B., and M.T. analyzed data; V.R., K.J.F., C. Sorg, and A.D. wrote the paper.

This work was supported by a university grant from the Kommission für klinische Forschung (Grant 8762754) at the Klinikum Rechts der Isar der Technischen Universität München to V.R. and A.D. We thank our colleagues at the Technischen Universität München-Neuroimaging Center, and in the departments of Neuroradiology and Nuclear Medicine, particularly Professor C. Zimmer, Professor M. Schwaiger, Dr. Igor Yakushev, Anne Winter, Sylvia Schachoff, Brigitte Dzewas, and Michael Herz.

${ }^{*}$ C.S. and A.D. contributed equally to this work.

The authors declare no competing financial interests.

Correspondence should be addressed to Valentin Riedl, Department of Neuroradiology, TUM-Neuroimaging Center, Klinikum Rechts der Isar der Technischen Universität München, Ismaninger Strasse 22, 81675 München, Germany.E-mail: valentin.ried@mytum.de.

DOI:10.1523/JNEUROSCI.0492-14.2014

Copyright $\odot 2014$ the authors $\quad 0270-6474 / 14 / 346260-07 \$ 15.00 / 0$ pinnings of rs-FC are still incompletely understood (Raichle and Mintun, 2006; Raichle, 2010). Resting-state electroencephalography (EEG) and magnetoencephalography (MEG) studies have found slow fluctuations in the power of alpha- and beta-band oscillations, which are correlated across remote brain areas, and such synchronized band-limited power fluctuations quantitatively link with BOLD rs-FC (Mantini et al., 2007; Brookes et al., 2011; Hipp et al., 2012). This means that in the resting-state local neuronal oscillations are synchronized by coherent power fluctuations on a systems level, and that these are spatially related to BOLD rs-FC.

These findings suggest a specific influence of the local activity (LA) of one area on FC at rest. Throughout systems neuroscience, one finds a recurrent theme that increasing the mean synaptic activity within a cortical area (or microcircuit) increases its sensitivity to afferent input from other areas. Examples of this association can be found at multiple scales: at the microscopic scale, simulations of integrate-and-fire neurons show that increased synchronization leads inevitably to increased synchronous gain and elevated mean firing rate levels (Chawla et al., 1999). This means that, at a microscopic scale, FC and mean activity levels are coupled in an obligatory fashion. At the macroscopic level, again using neuronally plausible simulations, the patterns of coherent large-scale BOLD fluctuations rest upon intercortical coupling of 
averaged ensemble activity and particularly of low-level spiking activity (Deco et al., 2013a,b). Only recently, this model has been supported by simultaneously acquired fMRI and full-band EEG data that found infraslow scalp potential fluctuations to be directly related to resting-state BOLD signals (Hiltunen et al., 2014).

In this article, we test the hypothesis that elevated mean neuronal activity levels are associated with increased coupling or FC among distributed cortical sources in the resting state. To address this hypothesis directly, we exploit recent advances in simultaneous multimodal neuroimaging, in which one can acquire direct [fluorodeoxyglucose (FDG)] measures of cerebral metabolism while measuring fluctuations in the BOLD signal reporting FC. These multimodal measurements are obtained using an integrated positron emission tomography (PET)/MRI scanner. Our design incorporates two different resting-state conditions (i.e., eyes closed and eyes open), allowing us to test the specificity of LA (i.e., how much of increased LA fuels FC).

\section{Materials and Methods}

Subjects. Twenty-four healthy subjects ( 16 males, 8 females; average age, $54.7 \pm 9.9$ years; all were right handed) participated in this simultaneous FDG-PET/fMRI brain imaging study, of whom 2 subjects had to be excluded during scanning (see below). Female participants ( $>40$ years of age) and male participants ( $>35$ years of age) were recruited from the greater Munich community, received an expense allowance, and gave informed consent to undergo procedures approved by the ethics review board of the Klinikum Rechts der Isar, Technische Universität München (TUM). Exclusion criteria included a history of psychiatric or neurological conditions, use of psychoactive medications, pregnancy and renal failure (critical for PET imaging), and common MR-related exclusion criteria.

Study design. We were interested in similarities and differences between the LA of FDG-PET data and the FC of the fMRI data that we simultaneously acquired during resting conditions with either eye closed (CLOSED) or with eyes open (OPEN). The amount of radiation exposure per single shot of FDG tracer prohibited repeated applications of FDG in the same subject. We therefore assigned subjects to either the CLOSED or OPEN group matched across age (CLOSED group, $56.9 \pm$ 9.9 years; OPEN group, $52.2 \pm 10.2$ years; $p=0.31$ ), sex (CLOSED group, three females; OPEN group, four females), and handedness (all were right handed).

We designed a resting-state scanning protocol that was suited equally well for both imaging techniques. In resting-state fMRI studies, resting conditions are usually limited to $\sim 10 \mathrm{~min}$ as subjects tend to quickly fall asleep during prolonged rest, which would then result in altered brain network configuration (Tagliazucchi et al., 2012). In FDG-PET imaging, the steady-state condition is maintained for at least $30 \mathrm{~min}$, during which time the tracer distributes equally across the entire brain (Fox et al., 1988; Drzezga et al., 2011). However, the most sensitive period in FDG imaging is the accumulation phase during the initial minutes after tracer injection when large amounts of FDG tracer have already accumulated in activated brain regions, depending on current glucose consumption (Vlassenko et al., 2006). To achieve the optimal signal-to-noise ratio, saturated images are acquired $30 \mathrm{~min}$ postinjection when the tracer is equally distributed across the entire brain and blood flow-dependent differences are rather negligible. This modified FDG-PET design of Vlassenko et al. (2006) is therefore ideally suited to achieve the best contrast between CLOSED and OPEN with both imaging techniques.

We started with an initial scanning period of 4 min, during which all subjects had to keep their eyes closed. This was followed by the functional CLOSED/OPEN run. Immediately before, we instructed subjects to keep their eyes closed/open, relax, and not to think of anything particular, but not to fall asleep. Together with a bolus injection of FDG tracer, we simultaneously started fMRI data acquisition. Immediately after the CLOSED/OPEN run, we interrogated the subjects about whether they had stayed awake. We had to exclude 2 subjects because they might have fallen asleep, thus reducing the image analysis to 22 subjects. Then all subjects kept their eyes closed again, and we performed structural imaging and diagnostic screening MR sequences. Thirty minutes postinjection, we recorded the saturated list mode PET dataset for $10 \mathrm{~min}$, reflecting FDG tracer distribution after homogeneous and complete distribution in the brain. All scanning was performed in a dimmed environment by switching off all lights, including those in the scanner bore. Light passing through the window of the console room was allowed to accurately perform FDG injection during MR scanning. Subjects had to fast during the $4 \mathrm{~h}$ before imaging. Immediately before scanning we performed a neuropsychiatric investigation to exclude current neurological or psychiatric diseases and explained the study protocol. We assessed the level of arousal and anxiety using the state-trait anxiety inventory in each subject before starting scanning (CLOSED group, $35.5 \pm 6.9$; OPEN group, $30.8 \pm 5.6 ; p=0.18$ ), and measured blood pressure and venous glucose levels $(<140 \mathrm{mg} / \mathrm{dl}$ for each subject). Subjects were prepared with a venous catheter to receive tracer injection during scanning while lying in the scanner bore.

Image acquisition. Scanning was performed on an integrated Siemens Biograph mMR scanner that was capable of simultaneously acquiring PET and MRI data using the vendor-supplied 12-channel phase-array head coil. PET images, magnetization prepared rapid acquisition gradient echo (MP-RAGE) T1-weighted anatomical images and $\mathrm{T} 2^{\star}$-weighted echoplanar imaging (EPI) MR data were acquired using the following scanning parameters: PET: list-mode acquisition, 128 slices (gap, 0.5 $\mathrm{mm}$ ) covering the whole brain; FOV, $450 \mathrm{~mm}$; matrix size, $192 \times 192$; voxel size, $3.7 \times 2.3 \times 2.7 \mathrm{~mm}^{3}$; EPI: TR, $2.000 \mathrm{~ms}$; TE, $30 \mathrm{~ms} /$ angle, $90^{\circ}$; 35 slices (gap, $0.6 \mathrm{~mm}$ ) aligned to $\mathrm{AC} / \mathrm{PC}$ covering the whole brain; FOV, $192 \mathrm{~mm}$; matrix size, $64 \times 64$; voxel size, $3.0 \times 3.0 \times 3.0 \mathrm{~mm}^{3}$ (each measurement consists of 300 acquisitions in interleaved mode with a total scan time of $10 \mathrm{~min}$ and $8 \mathrm{~s}$ ); MP-RAGE: TR, $2.300 \mathrm{~ms}$; TE, $2.98 \mathrm{~ms}$; angle, $9^{\circ}$; 160 slices (gap, $0.5 \mathrm{~mm}$ ) covering the whole brain; FOV, 256 $\mathrm{mm}$; matrix size, $256 \times 256$; voxel size, $1.0 \times 1.0 \times 1.0 \mathrm{~mm}^{3}$. The measurement has a total scan time of $5 \mathrm{~min}$ and $3 \mathrm{~s}$. During the initial 4 min of the design, we acquired arterial spin labeling data that were not analyzed for the current study.

Image preprocessing. DICOM (Digital Imaging and Communications in Medicine) images were first converted to 3D-NIFTI volumes and then were preprocessed with SPM8 (Wellcome Trust Center for Neuroimaging, London, UK). Preprocessing encompassed coregistration of the multimodal imaging dataset and preparation of the rs-fMRI EPI time series data for FC analysis separately for each subject. The first three images of each subject's EPI time series were deleted, and all remaining EPI images were realigned to this subject's mean EPI image using $6^{\circ}$ rigid-body transformation to correct for head motion during fMRI scanning. The mean FDG-PET image was first coregistered to the T1weighted image using rigid-body transformation, and both images were then coregistered to the mean EPI image, resulting in a subject-specific coregistered multimodal dataset. To allow for group analyses, each subject's brain data were then normalized to a standard template provided by the Montréal Neurological Institute (MNI) template using the SPM8 unified segmentation routine. MNI tissue-probability maps of gray matter, white matter, and CSF were warped onto single-subject T1-weighted images and were stored as masks for later use during the FC analysis. To achieve normalized single-subject data, the inverse transformation matrix of the warping process was later applied to T1-weighted, EPI, and PET images, and all EPI and PET images were resampled to an isotropic voxel size of $3 \times 3 \times 3 \mathrm{~mm}^{3}$. Finally, PET images were scaled by normalization of whole-brain FDG uptake values and were spatially smoothed using a Gaussian kernel full-width at half-maximum of $12 \mathrm{~mm}$.

Next, a series of FC-specific preprocessing steps were applied to the rs-EPI volumes as previously discussed (Van Dijk et al., 2010) using the Data Processing Assistant for Resting-State fMRI (DPARSF) toolbox (Chao-Gan and Yu-Feng, 2010). We performed slice-time correction to account for differences in slice acquisition time and removed a series of nuisance regressors reflecting spurious noise or systematic variance associated with non-neuronal sources using partial regression. Nuisance regressors included the six motion vectors computed during the rigidbody head motion correction, and two vectors with averaged white mat- 
Table 1. Brain regions with increased $\mathrm{LA}$, increased $\mathrm{FC}$, and overlapping increases in $\mathrm{LA}$ and $\mathrm{FC}$

\begin{tabular}{|c|c|c|c|c|c|}
\hline \multirow[b]{2}{*}{ Region } & \multirow{2}{*}{$\begin{array}{l}\text { Center of mass } \\
(x, y, z \text { coordinates in MNI space) }\end{array}$} & \multirow[b]{2}{*}{ PET statistics } & \multicolumn{2}{|c|}{ fMRI statistics } & \multirow{2}{*}{$\begin{array}{l}\text { Cluster size } \\
\text { overlap (voxels) }\end{array}$} \\
\hline & & & V1 seed & $\overline{\text { SAL seed }}$ & \\
\hline \multirow[t]{3}{*}{ Occipital cortex: calcarine, lingual, fusiform gyrus } & $15,-74,7$ & 14.75 & & & 181 \\
\hline & $-15,-99,9$ & & & 4.57 & \\
\hline & $9,-81,9$ & & & 4.82 & \\
\hline Extrastriate cortex & $-41,-81,19$ & 10.19 & & & \\
\hline Cuneus & $18,-79,31$ & 8.58 & & & \\
\hline $\mathrm{mCC}$ & $0,-31,37$ & 11.81 & & & 212 \\
\hline \multirow{2}{*}{ Left al } & $-51,11,7$ & 8.85 & & & 211 \\
\hline & $-42,15,-3$ & & 7.03 & & \\
\hline Left inferior frontal gyrus & $-48,29,-2$ & 8.84 & & & \\
\hline Left superior frontal gyrus & $-30,66,6$ & & 5.89 & & \\
\hline Right superior frontal gyrus & $24,63,-6$ & & 6.81 & & \\
\hline Middle superior frontal gyrus & $-6,50,37$ & 10.6 & & & \\
\hline \multirow[t]{2}{*}{ Right al } & $36,14,4$ & 8.64 & & & 284 \\
\hline & $51,21,-6$ & & 6.22 & & \\
\hline Fusiform/inferior temporal gyrus & $36,-10,-38$ & 7.94 & & & \\
\hline Thalamus & $-3,-24,6$ & & 5.71 & & \\
\hline Cerebellum & $36,-54,-27$ & & 8.55 & & \\
\hline Brainstem & $-3,-21,-18$ & & 6.42 & & \\
\hline
\end{tabular}

Data are $t$ statistics, unless otherwise indicated. Bold indicates overlapping increases in LA and FC. See also Figure 1 and main text.

ter and CSF signals derived from masks generated during the unified segmentation process. We did not regress out the global gray matter signal as ongoing discussions have provided suspicion that anticorrelations might be artificially revealed in resting-state FC analyses (Van Dijk et al., 2010). We finally applied temporal bandpass filtering to retain frequencies between 0.01 and $0.08 \mathrm{~Hz}$, and spatial smoothing using a Gaussian kernel full-width at half-maximum of $8 \mathrm{~mm}$.

Functional connectivity analysis and group statistics. LA and FC during CLOSED and OPEN conditions were identified using a general linear model and Gaussian random field theory (RFT) as implemented in SPM8. We derived whole-brain group statistical parametric maps (SPMs) of changes in LA by subjecting each participant's FDG image to a second-level random-effects model. In a whole-brain analysis of covariance (ANCOVA) of FDG-PET data correcting for grand mean FDG uptake, we calculated contrasts CLOSED > OPEN and OPEN > CLOSED, and applied a familywise error (FWE)-corrected $p$ value of $<0.05$ on the voxel level using Gaussian RFT to correct for multiple testing. For illustrations, each SPM was projected on a single-subject high-resolution structural brain image derived from MRIcro (Chris Rorden, McCausland Center for Brain Imaging, Columbia, SC).

Calculation of FC maps was performed again using the DPARSF toolbox. For each subject, we created whole-brain FC maps by entering an averaged time series across all voxels of a seed region as the variable of interest in a linear regression with the time series of each voxel across the entire brain. The seed regions of interest (ROIs) were derived from the independent second-level FDG-PET analysis, revealing a visual and salience (SAL) network. To determine the most representative voxels of each network, we applied a more stringent threshold during the FDG analysis $\left(p<5 \times 10^{-6}\right)$, and created separate seed time series averaged across all voxels in primary visual cortex (V1) and the SAL network regions. We derived whole-brain group SPMs of changes in FC by subjecting each participant's Fisher $z$-transformed FC map to a second-level random-effects model. We calculated voxelwise independent-samples $t$ tests for contrasts CLOSED > OPEN and OPEN > CLOSED, and applied a FWE-corrected $p$ value of $<0.05$ on the cluster level with $p<$ 0.001 as the height threshold. To visualize the spatial overlap between changes in local and network activity, we projected both group FDGSPMs and FC-SPMs as overlays on a structural brain image and determined the intersection of both modalities, defined as those voxels contained in 2/2 maps at these thresholds, resulting in clusters of $\sim 200$ voxels (Table 1, peak coordinates and cluster size).

We calculated the spatial similarity (SpaSi) coefficient (i.e., the relationship between LA and FC in a certain ROI) as the voxelwise spatial correlation coefficient between normalized LA and FC values in this ROI (SpaSi[LA,FC]). For each subject, we calculated SpaSi[LA,V1-FC] ROI for all SAL ROIs anterior insulae (aI), anterior (aCC), middle cingulate (mCC), and SpaSi[LA,SAL-FC](V1) for V1 ROI, and entered these into a two-way ANOVA testing for condition (CLOSED, OPEN) and ROI (V1, aI, aCC, mCC) effects. Post hoc tests were performed with independentsamples $t$ tests.

\section{Results}

We performed simultaneous resting-state FDG-PET and fMRI scanning in healthy human subjects $(n=22$, age, $54.6 \pm 10.2$ years; females, 7; males, 15), and investigated the relationship between LA (FDG) and FC (BOLD fMRI) in two brain states corresponding to CLOSED $(n=11)$ and $\operatorname{OPEN}(n=11)$ restingstate conditions.

\section{FDG-PET analysis}

Figure $1 A$ shows the voxelwise FDG-uptake during CLOSED and OPEN conditions (ANCOVA, FWE corrected, $p<0.05$ ) corrected for whole-brain uptake values. In the cerebral cortex, glucose metabolism was highest in medial occipital [Brodmann areas (BAs) 17-19], parietal (BAs 1-3, 7, and 40), frontal (BAs 9 and 25), inferior temporal cortex (BA 20), middle cingulate cortex (BA 23), and superior cerebellar lobes. Analyzing changes in LA between CLOSED and OPEN conditions, the whole-brain ANCOVA revealed increased $\mathrm{LA}_{\text {OPEN }>\text { CLOSED }}$ in the occipital lobe, comprising V1 and secondary visual cortices (together termed the VIS network) and in areas known to be part of the SAL network (Seeley et al., 2007), comprising bilateral\& aI, aCC, and mCC (FWE corrected, $p<0.05$; Fig. 2A; Table 1). We found no significant increase of LA for the opposite contrast of LA $_{\text {CLOSED }>\text { OPEN }}$. 

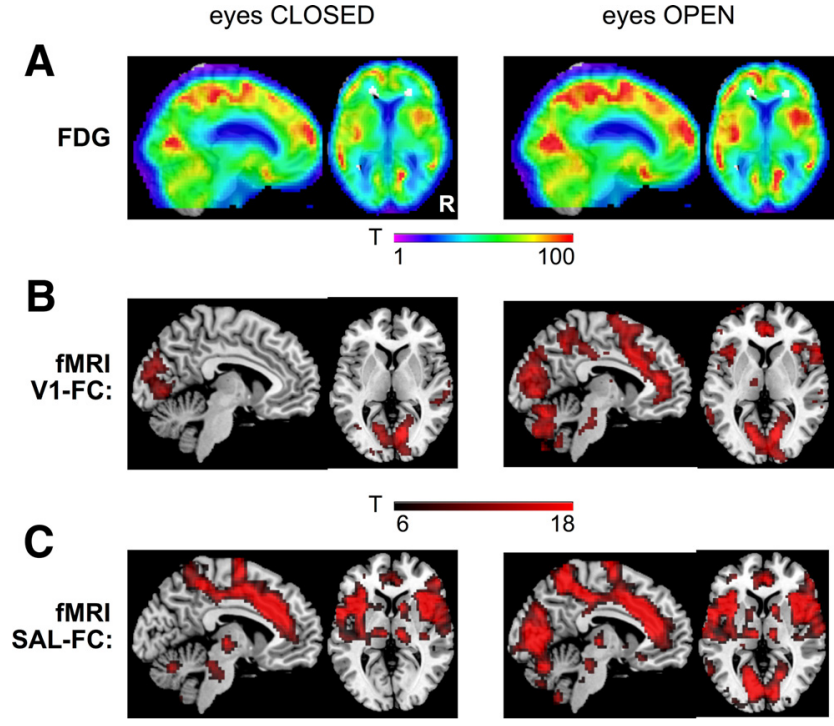

$x=102, z=76$

Figure 1. LA and FC during resting-state CLOSED (left) and OPEN (right) conditions. A, SPMs of whole-brain voxelwise one sample $t$ tests for FDG uptake controlling for each subject's grand mean signal. $\boldsymbol{B}$, SPMs of whole-brain voxelwise one-sample $t$ tests on individual FC maps revealing rs-FC for seed ROI V1 (V1-FC). Regions (red) with V1-FC during the CLOSED condition include primary and secondary visual cortex, and inferior parietal and temporoparietal areas. Additional regions with V1-FC during the OPEN condition include cingulate, insular, and prefrontal cortices; brainstem; thalamus; and cerebellum. C, SPMs of whole-brain voxelwise one sample $t$ tests on individual $\mathrm{FC}$ maps revealing rs- $\mathrm{FC}$ for averaged seed ROI of the SAL network (SAL-FC). Regions (red) with SAL-FC during the CLOSED condition include cingulate, insular, superior parietal, and prefrontal cortices; brainstem; thalamus; and cerebellum. Additional regions with SAL-FC during the OPEN condition included primary and secondary visual, and anterior cingulate cortices. All SPMs are thresholded at an FWE-corrected $p$ value of $<0.05$ and are superimposed on a high-resolution T1-weighted anatomical image. $x, z$, Coordinates of brain slices in MNI space; $R$, right side of the brain.

\section{Rs-fMRI analysis}

Next, we analyzed FC within and between the VIS and SAL networks on the independent fMRI data that were acquired at the same time as the measurement of LA. For each subject, we extracted an averaged BOLD signal time series summarizing the activity of the VIS and SAL networks and calculated whole-brain FC using voxelwise linear regression analyses. Specifically, we identified the peak clusters of each network from the FDG-PET analysis and averaged the BOLD signal over $\mathrm{V} 1$ and combined SAL network regions ( $\mathrm{aCC}, \mathrm{mCC}$, and aI; for cluster information, see Table 1). During CLOSED conditions, one-sample $t$ tests (FWE corrected, $p<0.05$ ) revealed strictly separated withinnetwork FC of the visual and salience networks, as described in previous studies (Deen et al., 2011; Yeo et al., 2011). Particularly,

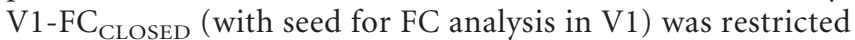
to primary and secondary visual cortices (Fig. $1 B$, left), and SAL- $\mathrm{FC}_{\mathrm{CLOSED}}$ was found in aCC, $\mathrm{mCC}$, aI, bilateral thalamus, brainstem, and cerebellum (Fig. 1C, left). However, during the OPEN condition, we observed additional between-network coupling (Fig. $1 B, C$, right), which was quantified in Independentsamples $t$ tests (FWE corrected, $p<0.05$; Fig. 2B). V1$\mathrm{FC}_{\mathrm{OPEN}>\mathrm{CLOSED}}$ was significantly increased to all SAL regions (i.e., bilateral aI, aCC, mCC, thalamus, brainstem, and cerebellum). The SAL-FC $\mathrm{OPEN}_{\mathrm{OCLOSED}}$ was selectively increased to primary and secondary visual areas and aCC. Again, we found no significant $\mathrm{FC}_{\mathrm{CLOSED}>\mathrm{OPEN}}$ for the opposite contrast for either V1-FC or SAL-FC.

\section{Integrated FDG/fMRI analysis}

Critically, the conjunction analysis in Figure $2 C$ illustrates the remarkable overlap between increased $\mathrm{LA}_{\mathrm{OPEN}}>\mathrm{CLOSED}$ and $\mathrm{FC}_{\mathrm{OPEN}>\mathrm{CLOSED}}$ in $\mathrm{V} 1$ and all major SAL regions (i.e., aI, aCC, and mCC; for cluster sizes and centers of mass, see Table 1). This spatial overlap provides first evidence for the specific relevance of increased LA for the emergence of FC. To specify the link between LA and FC, we investigated whether a direct relationship between LA and FC exists at a finer spatial level. We defined an SpaSi coefficient as the voxelwise spatial correlation between normalized LA and FC values within each of the overlapping ROIs (SpaSi[LA,FC] ROI). SpaSi quantitatively reflects the amount of LA dedicated to FC. An example of the first subject's voxel values of LA(aI) and V1-FC(aI) can be seen in Figure 2C. Although we had smoothed the data, the spatial correlations included relatively large ROIs (e.g., the insula ROI included 284 voxels). In the following, we report SpaSi results for (1) between-network FC and (2) within-network FC.

\section{SpaSi of between-network FC}

For each subject, we calculated the SpaSi of LA and betweennetwork FC separately for $\mathrm{V} 1, \mathrm{aCC}, \mathrm{mCC}$, and aI, and entered these into two-way ANOVA testing for condition (CLOSED, OPEN) and ROI (V1, aI, aCC, mCC) effects. Between-network FC is defined as the FC between VIS and SAL (i.e., V1-FC to SAL regions and vice versa). The ANOVA revealed significant main effects for condition and ROI (condition: $F_{(1,20)}=20.9, p=1.8 \times$ $10^{-4}$; ROI: $\left.F_{(3,18)}=25.5, p=1 \times 10^{-6}\right)$ and a significant interaction $\left(F_{(6,15)}=3.5, p=0.039\right)$. This indicates that $\mathrm{SpaSi}_{\mathrm{CLOSED}}$ and $\mathrm{SpaSi}_{\text {OPEN }}$ significantly differ, and this effect depends on the region. Post hoc independent-samples $t$ tests revealed increased SpaSi during the OPEN condition in $\mathrm{V} 1$, aI, and aCC $\left(\mathrm{V} 1: t_{(20)}=\right.$ $4.2, p=4 \times 10^{-4}$; aI: $t_{(20)}=3.3, p=0.004$; aCC: $t_{(20)}=4.4, p=$ $3 \times 10^{-4}$; Fig. $2 D$ ). For all three ROIs, one-sample $t$ tests also showed a significant correlation of LA and between-network FC profiles only for $\mathrm{SpaSi}_{\mathrm{OPEN}}$ (V1: $t_{(10)}=11.9, p=3 \times 10^{-7}$; aI: $t_{(10)}=-3.1, p=0.01$; aCC: $\left.t_{(10)}=8.7, p=6 \times 10^{-6}\right)$ but not for $\operatorname{SpaSi}_{\text {CLOSED }}\left(\mathrm{V} 1: t_{(10)}=2.1, p=0.06 ; \mathrm{aI}: t_{(10)}=-1.8, p=0.1\right.$; aCC: $\left.t_{(10)}=-0.5, p=0.6\right)$. No difference was found in mCC $\left(t_{(20)}=-0.4, p=0.7\right)$. This indicates that LA in key regions of both networks significantly contributes to the between-network FC of this region during the OPEN condition, which is absent during the CLOSED condition. We also quantified the percentage of LA explaining between-network FC. The mean square SpaSi coefficients in Figure $2 E$ illustrate the increased similarity in regional variance of LA and between-network FC. As an example, the LA profile in V1 explains $29 \%$ of FC with the SAL network that emerges only during the OPEN condition.

\section{SpaSi of within-network FC}

Beyond between-network FC, we also analyzed LA effects on within-network FC in the VIS network by calculating SpaSi [LA (V1-FC)] for all voxels of the VIS network as defined in Figure $1 B$, left. An independent-samples $t$ test revealed no difference between $\operatorname{SpaSi}_{\text {CLOSED }}$ and $\operatorname{SpaSi}_{\text {OPEN }}\left(t_{(20)}=1.5, p=0.2\right)$. However, LA and within-network FC in the VIS-network were highly correlated during both conditions $\left(\mathrm{SpaSi}_{\mathrm{CLOSED}}: t_{(10)}=17.1, p=\right.$ $9 \times 10^{-7} ; \mathrm{SpaSi}_{\mathrm{OPEN}}: t_{(10)}=38.1, p=4 \times 10^{-12} ;$ Fig. $\left.2 D\right)$. This means that LA in visual areas determines FC within the VIS network during both the eyes closed and eyes open conditions. Again, the mean square SpaSi coefficients in Figure 2E quantify this relationship. During both conditions, LA in V1 continuously explains between $44 \%$ and $52 \%$ of FC with the VIS network. 


\section{Discussion}

Our findings reveal that regional activity contributes significantly toward explaining BOLD FC within functional systems in the resting state, and that increased LA during simple state changes contributes to BOLD FC between functional systems. We establish the specificity of this association-both in terms of brain states and in terms of spatial profiles. For example, we show that the FC between visual cortical areas and the salience network increases in an eyes open condition relative to an eyes closed condition. Crucially, the pattern of elevated FC coincides almost exactly with the spatial pattern of increased regional activity. At a finer spatial level, we also established a correlation, over voxels, between mean activity profiles and patterns of FC within large regions of interest. In short, we provide empirical evidence- - using conjoint measures of local and distributed dynamicsthat local activity determines global dynamics at rest-as would be expected on the basis of theoretical and computational considerations.

Our results have several implications for the interpretation of rs-FC from BOLD fMRI data. First, we provide evidence for the neuronal basis of BOLD FC on the voxel level. Combined EEG/fMRI and MEG/fMRI studies have revealed neurophysiological correlates of rs-FC patterns on the systems level, particularly correlations between BOLD signal and power fluctuations in alpha and beta bands across distant brain areas (Nir et al., 2008; Sadaghiani et al., 2010). Here, we reveal a relationship between voxelwise measures of LA (as measured with FDGPET) and BOLD FC within and between functional networks. Specifically, LA in primary visual areas strongly determines FC within the visual network independent of the brain state, supporting recent findings of LA-FC coupling in separately acquired human data (Tomasi et al., 2013) and in simultaneous recordings in animals (Wehrl et al., 2013). In addition to this consistency of LA-FC coupling within the visual network independent of brain state, we also found state-related increases of LA determining changes of between-network FC. While our analysis was restricted to only two functional networks and their interaction, future applications of the SpaSi approach might reveal a complete coverage of FC changes related to increased LA in a certain voxel.

Second, we offer a voxelwise approach to integrate multimodal imaging parameters that might allow quantifying the relationship between structural and functional connectivity. Structural connectivity patterns seem to determine FC patterns to a large extent (Honey et al., 2009; van den Heuvel and Sporns, 2013; Hinne et al., 2014) and particularly at a critical working can be found in Table 1.
B
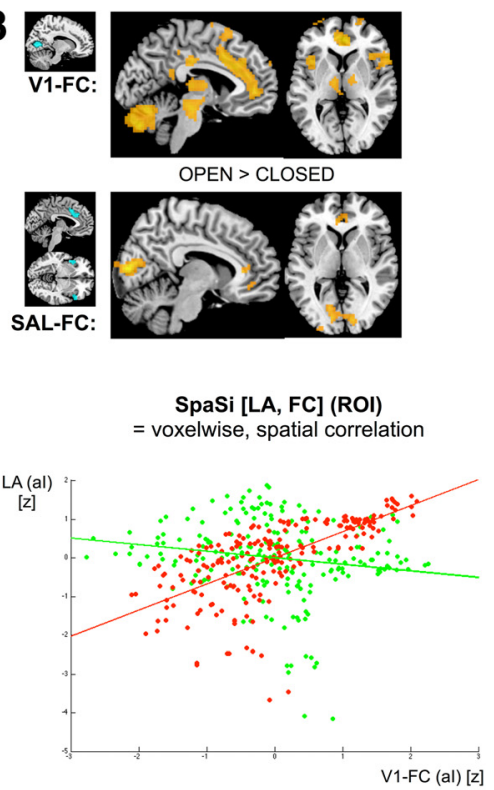

E

\begin{tabular}{|c|c|c|}
\hline$\underset{\left(\text { mean } r^{2}\right)}{\operatorname{SpaSi}}$ & CLOSED & OPEN \\
\hline \multicolumn{3}{|c|}{ between-network FC } \\
\hline V1 [SAL-FC] & 0.02 & $0.29 * * *$ \\
\hline $\operatorname{aCC}_{[\mathrm{V} 1-\mathrm{FC}]}$ & 0.002 & $0.21 * * *$ \\
\hline al $[\mathrm{V} 1-\mathrm{FC}]$ & 0.01 & 0.04 * \\
\hline \multicolumn{3}{|c|}{ within-network FC } \\
\hline VIS $[\mathrm{V} 1-\mathrm{FC}]$ & $0.44^{* \star *}$ & $0.52^{* \star *}$ \\
\hline
\end{tabular}

Figure 2. Quantitative relationship between $L A$ and FC in the resting human brain. $A$, SPMs of whole-brain voxelwise ANCOVA for LA changes controlling for each subject's grand mean signal. Regions (cyan) with increased LA during the OPEN condition included V1, al, aCC, and mCC. FWE corrected, $p<0.05$. R, Right. $\boldsymbol{B}$, SPMs of whole-brain voxelwise independent-samples $t$ tests on individual FC maps for seed R0ls (cyan) V1 (V1-FC; top row) and SAL hubs (SAL-FC; bottom row) revealing FC changes. Regions with increased SAL-FC during the OPEN condition was V1 and parts of aCC. $p<0.05$, FWE corrected. C, Voxelwise conjunction showing regions (violet) with increased LA and FC. An exemplary distribution of voxel values of LA ( $y$-axis) and V1-FC ( $x$-axis) of al FC] ROI coefficient for each subject and each ROI as the voxelwise spatial correlation between normalized LA and between-network values, and subsequently analyzed them in a two-way ANOVA. D, Post hoc independent-samples $t$ tests revealed significantly

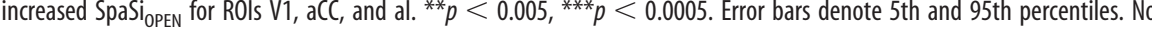
(

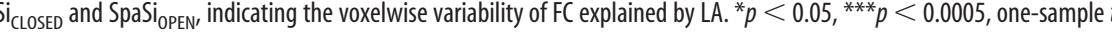
tests performed separately for $\mathrm{SpaSi}_{\mathrm{CLOSED}}$ and $\mathrm{SpaSi}_{\mathrm{OPEN}}$. Cluster coordinates for all SPMs and cluster sizes for conjunction regions

point (Deco et al., 2013b). While those structural parameters tend to be highly consistent across time, BOLD FC patterns may vary across behavioral states and therefore may not exclusively reflect anatomical connections (Albert et al., 2009; Lewis et al., 2009; Riedl et al., 2011; Harmelech et al., 2013). The SpaSi coefficient may therefore be suitable to quantify portions of FC related to structural determinants such as structural connectivity from diffusion-weighted imaging or gray matter density.

Third, the multimodal investigation of brain states with both LA and BOLD FC might shed light on an ongoing discussion about the neuronal basis of anticorrelated FC that has been observed between certain FC networks and under certain conditions (Fox et al., 2009; Chai et al., 2012; Keller et al., 2013). While some studies suggest that anticorrelated BOLD FC is a method- 
ological byproduct of regressing the fMRI time series against the global signal of the fMRI volume, others found evidence for a neurophysiological origin of anticorrelated BOLD signal fluctuations. We suggest that the simultaneous acquisition and analysis of local neuronal activity and rs-FC under conditions known to induce anticorrelated FC might answer whether anticorrelated FC is indeed associated with decreased neuronal activity.

Fourth, the simultaneous acquisition of LA and BOLD FC might serve as a model setup for clinical neuroscience. An extensive amount of literature has been reporting on rs-FC changes in neuropsychiatric patients with neurodegenerative diseases and affective disorders (Greicius, 2008; Menon, 2011). For several of these patient groups, region-specific changes in LA have also been reported, but these measures have not yet been integrated. Therefore, any metabolic or neurotransmitter changes related to BOLD FC changes are still largely unknown. Here, simultaneous PET/ fMRI imaging with PET tracers like FDG or neurotransmitter tracers associated with dopamine or glutamate might reveal neurophysiological correlates of dysconnectivity patterns observed in neuropsychiatric diseases and their associations or dissociations with metabolic changes of glucose or neurotransmitter metabolism.

There are also several limitations of our study that restrict the interpretation of the data. While we found robust increases of LA and FC between eyes closed and open conditions, the reason for these local and systemic changes remains unclear. Although participants had eyes closed and open in a heavily dimmed scanner environment, effects might be driven by preparatory brain state changes or partial visual input. However, recent data suggest that, surprisingly, visual input plays a minor role in explaining these brain signal changes. Using a $2 \times 2$ design of eyes closed/open in dark and illuminated environments, a recent study (Jao et al., 2013) found experimental evidence for brain state changes having the most influence on functional network reconfiguration, while the effect of visual input was negligible. Moreover, computational data suggest that rs-FC is the "optimal working point for incoming sensory information" from an "ecological and economical" point of view (Deco et al., 2013a). However, further studies need to specifically explore these effects in a multimodal imaging setting.

Finally, to reduce the radioactive exposure of our participants, we did not implement a crossover design but rather allocated each subject to one of the two conditions. Therefore, with our current data we are not able to quantify the within-subject increase of LA and rs-FC with respect to state changes. On the other hand, taking the higher between-subject variance into account our data suggest even tighter coupling between LA and rs-FC in repeated-measures designs. Moreover, absolute quantification of glucose consumption was not possible as equipment to sample arterial blood in the MR environment has been developed only recently; therefore, direct estimation of individual input functions to achieve quantitative PET measurement will only be available for future measurements.

\section{Conclusion}

Computational and animal data suggest close interaction between local neuronal activity and the generation of FC from BOLD fMRI data. In a simultaneous imaging setup of FDG-PET scanning and fMRI, we provide the first evidence in humans that local energy consumption contributes to functional coupling within and across brain networks. Specifically, voxelwise baseline metabolism within the visual system was consistently related to FC of the visual system during both the eyes closed and open conditions. Additionally, increased levels of metabolism during the eyes open condition contributed to increased FC between visual and salience networks. Our data support computational models suggesting that synchronized local dynamics give rise to large-scale networks of BOLD FC.

\section{References}

Albert NB, Robertson EM, Miall RC (2009) The resting human brain and motor learning. Curr Biol 19:1023-1027. CrossRef Medline

Bressler SL, Menon V (2010) Large-scale brain networks in cognition: emerging methods and principles. Trends Cogn Sci 14:277-290. CrossRef Medline

Brookes MJ, Woolrich M, Luckhoo H, Price D, Hale JR, Stephenson MC, Barnes GR, Smith SM, Morris PG (2011) Investigating the electrophysiological basis of resting state networks using magnetoencephalography. Proc Natl Acad Sci U S A 108:16783-16788. CrossRef Medline

Chai XJ, Castañón AN, Ongür D, Whitfield-Gabrieli S (2012) Anticorrelations in resting state networks without global signal regression. Neuroimage 59:1420-1428. CrossRef Medline

Chao-Gan Y, Yu-Feng Z (2010) DPARSF: a MATLAB Toolbox for "Pipeline" data analysis of resting-state fMRI. Front Syst Neurosci 4:13. CrossRef Medline

Chawla D, Lumer ED, Friston KJ (1999) The relationship between synchronization among neuronal populations and their mean activity levels. Neural Comput 11:1389-1411. CrossRef Medline

Deco G, Jirsa VK, McIntosh AR (2013a) Resting brains never rest: computational insights into potential cognitive architectures. Trends Neurosci 36:268-274. CrossRef Medline

Deco G, Ponce-Alvarez A, Mantini D, Romani GL, Hagmann P, Corbetta M (2013b) Resting-state functional connectivity emerges from structurally and dynamically shaped slow linear fluctuations. J Neurosci 33:1123911252. CrossRef Medline

Deen B, Pitskel NB, Pelphrey KA (2011) Three systems of insular functional connectivity identified with cluster analysis. Cereb Cortex 21:1498-1506. CrossRef Medline

Drzezga A, Becker JA, Van Dijk KR, Sreenivasan A, Talukdar T, Sullivan C, Schultz AP, Sepulcre J, Putcha D, Greve D, Johnson KA, Sperling RA (2011) Neuronal dysfunction and disconnection of cortical hubs in nondemented subjects with elevated amyloid burden. Brain 134:1635-1646. CrossRef Medline

Fox MD, Raichle ME (2007) Spontaneous fluctuations in brain activity observed with functional magnetic resonance imaging. Nat Rev Neurosci 8:700-711. CrossRef Medline

Fox MD, Zhang D, Snyder AZ, Raichle ME (2009) The global signal and observed anticorrelated resting state brain networks. J Neurophysiol 101: 3270-3283. CrossRef Medline

Fox PT, Raichle ME, Mintun MA, Dence C (1988) Nonoxidative glucose consumption during focal physiologic neural activity. Science 241:462464. CrossRef Medline

Greicius M (2008) Resting-state functional connectivity in neuropsychiatric disorders. Curr Opin Neurol 21:424-430. CrossRef Medline

Harmelech T, Preminger S, Wertman E, Malach R (2013) The day-after effect: long term, Hebbian-like restructuring of resting-state fMRI patterns induced by a single epoch of cortical activation. J Neurosci 33:94889497. CrossRef Medline

Hiltunen T, Kantola J, Abou Elseoud A, Lepola P, Suominen K, Starck T, Nikkinen J, Remes J, Tervonen O, Palva S, Kiviniemi V, Palva JM (2014) Infra-slow EEG fluctuations are correlated with resting-state network dynamics in fMRI. J Neurosci 34:356-362. CrossRef Medline

Hinne M, Ambrogioni L, Janssen RJ, Heskes T, van Gerven MA (2014) Structurally-informed Bayesian functional connectivity analysis. Neuroimage 86:294-305. CrossRef Medline

Hipp JF, Hawellek DJ, Corbetta M, Siegel M, Engel AK (2012) Large-scale cortical correlation structure of spontaneous oscillatory activity. Nat Neurosci 15:884-890. CrossRef Medline

Honey CJ, Sporns O, Cammoun L, Gigandet X, Thiran JP, Meuli R, Hagmann P (2009) Predicting human resting-state functional connectivity from structural connectivity. Proc Natl Acad Sci US A 106:2035-2040. CrossRef Medline

Jao T, Vértes PE, Alexander-Bloch AF, Tang IN, Yu YC, Chen JH, Bullmore ET (2013) Volitional eyes opening perturbs brain dynamics and func- 
tional connectivity regardless of light input. Neuroimage 69:21-34. CrossRef Medline

Keller CJ, Bickel S, Honey CJ, Groppe DM, Entz L, Craddock RC, Lado FA, Kelly C, Milham M, Mehta AD (2013) Neurophysiological investigation of spontaneous correlated and anticorrelated fluctuations of the BOLD signal. J Neurosci 33:6333-6342. CrossRef Medline

Lewis CM, Baldassarre A, Committeri G, Romani GL, Corbetta M (2009) Learning sculpts the spontaneous activity of the resting human brain. Proc Natl Acad Sci U S A 106:17558-17563. CrossRef Medline

Mantini D, Perrucci MG, Del Gratta C, Romani GL, Corbetta M (2007) Electrophysiological signatures of resting state networks in the human brain. Proc Natl Acad Sci U S A 104:13170-13175. CrossRef Medline

Menon V (2011) Large-scale brain networks and psychopathology: a unifying triple network model. Trends Cogn Sci 15:483-506. CrossRef Medline

Nir Y, Mukamel R, Dinstein I, Privman E, Harel M, Fisch L, Gelbard-Sagiv H, Kipervasser S, Andelman F, Neufeld MY, Kramer U, Arieli A, Fried I, Malach R (2008) Interhemispheric correlations of slow spontaneous neuronal fluctuations revealed in human sensory cortex. Nat Neurosci 11:1100-1108. CrossRef Medline

Raichle ME (2010) Two views of brain function. Trends Cogn Sci 14:180190. CrossRef Medline

Raichle ME, Mintun MA (2006) Brain work and brain imaging. Annu Rev Neurosci 29:449-476. CrossRef Medline

Riedl V, Valet M, Wöller A, Sorg C, Vogel D, Sprenger T, Boecker H, Wohlschläger AM, Tölle TR (2011) Repeated pain induces adaptations of intrinsic brain activity to reflect past and predict future pain. Neuroimage 57:206-213. CrossRef Medline

Sadaghiani S, Scheeringa R, Lehongre K, Morillon B, Giraud AL, Kleinschmidt A (2010) Intrinsic connectivity networks, alpha oscillations, and tonic alertness: a simultaneous electroencephalography/functional magnetic resonance imaging study. J Neurosci 30:10243-10250. CrossRef Medline

Seeley WW, Menon V, Schatzberg AF, Keller J, Glover GH, Kenna H, Reiss AL, Greicius MD (2007) Dissociable intrinsic connectivity networks for salience processing and executive control. J Neurosci 27:2349-2356. CrossRef Medline

Tagliazucchi E, von Wegner F, Morzelewski A, Borisov S, Jahnke K, Laufs H (2012) Automatic sleep staging using fMRI functional connectivity data. Neuroimage 63:63-72. CrossRef Medline

Tomasi D, Wang GJ, Volkow ND (2013) Energetic cost of brain functional connectivity. Proc Natl Acad Sci U S A 110:13642-13647. CrossRef Medline

van den Heuvel MP, Sporns O (2013) An anatomical substrate for integration among functional networks in human cortex. J Neurosci 33:1448914500. CrossRef Medline

Van Dijk KR, Hedden T, Venkataraman A, Evans KC, Lazar SW, Buckner RL (2010) Intrinsic functional connectivity as a tool for human connectomics: theory, properties, and optimization. J Neurophysiol 103:297-321. CrossRef Medline

Vlassenko AG, Rundle MM, Mintun MA (2006) Human brain glucose metabolism may evolve during activation: findings from a modified FDG PET paradigm. Neuroimage 33:1036-1041. CrossRef Medline

Wehrl HF, Hossain M, Lankes K, Liu CC, Bezrukov I, Martirosian P, Schick F, Reischl G, Pichler BJ (2013) Simultaneous PET-MRI reveals brain function in activated and resting state on metabolic, hemodynamic and multiple temporal scales. Nat Med 19:1184-1189. CrossRef Medline

Yeo BT, Krienen FM, Sepulcre J, Sabuncu MR, Lashkari D, Hollinshead M, Roffman JL, Smoller JW, Zöllei L, Polimeni JR, Fischl B, Liu H, Buckner RL (2011) The organization of the human cerebral cortex estimated by intrinsic functional connectivity. J Neurophysiol 106:1125-1165. CrossRef Medline 